\title{
Los betabloqueantes no son fármacos de primera línea en el tratamiento de la hipertensión arterial
}

Beta-blockers are not first-line treatment drugs in hypertension

Wiysonge CS y col. Cochrane Database of Systematic Reviews (2017), №1. Art. No:CD002003

\section{Objetivos}

Evaluar los efectos de los betabloqueantes (BB) sobre la morbilidad (eventos cerebrovasculares y cardiovasculares) y mortalidad en adultos con hipertensión arterial (HTA).

\section{Fuente de datos}

Se revisaron el Registro Cochrane Especializado en HTA, el Registro Cochrane CENTRAL de Ensayos Controlados, MEDLINE (desde 1946), EMBASE (desde 1974) y el registro de ensayos ClinicalTrials.gov, en busca de ensayos clínicos controlados aleatorizados (ECA) hasta junio de 2016. Además se realizó una búsqueda en la Plataforma de registro de ensayos clínicos internacionales de la Organización Mundial de la Salud (Julio 2015).

\section{Selección de estudios}

Se incluyeron ECA, de al menos un año de seguimiento, que evaluaran los efectos de los BB sobre morbilidad y mortalidad en adultos hipertensos de al menos 18 años de edad, en comparación con placebo $u$ otras drogas, como tratamiento de primera línea para la HTA. Los estudios debían reportar además eventos adversos.

\section{Extracción de datos}

Los datos fueron extraídos por dos revisores independientes quienes analizaron el riesgo de sesgos y resolvieron las discrepancias por consenso. Las medidas de resultado fueron las variables de morbilidad, mortalidad y eventos adversos. Se analizaron los resultados según intención de tratar y se expresaron como riesgo relativo (RR) con sus intervalos de confianza del 95\% (IC95\%). Asimismo utilizaron la escala de GRADE para valorar la calidad de la evidencia.

\section{Resultados principales}

Se incluyeron 13 ensayos (91.561 participantes) que compararon BB con: placebo (4 estudios, 23.613 participantes), diuréticos (5 estudios, 18.241 participantes), calcio-antagonistas (4 estudios, 44.825 participantes), inhibidores del sistema reninaangiotensina-aldosterona (3 estudios, 10.828 participantes). Muchos ensayos tuvieron alto riesgo de sesgo y el $75 \%$ de los participantes recibieron atenolol. No se encontraron estudios con BB vasodilatadores (por ejemplo, nebivolol) o que evaluaran los efectos en jóvenes y ancianos.

Los resultados principales se muestran en la Tabla 1.

Tabla 1. Comparación de beta-bloqueantes (BB) versus placebo y otros antihipertensivos como tratamiento de primera línea en hipertensión arterial.

\begin{tabular}{|c|c|c|c|c|}
\hline Resultado & Placebo & Diuréticos & Calcio-antagonistas & Inhibidores del SRAA \\
\hline & $\begin{array}{c}\text { RR } \\
\text { (IC95\%) }\end{array}$ & $\begin{array}{c}\text { RR } \\
\text { (IC95\%) }\end{array}$ & $\begin{array}{c}\text { RR } \\
\text { (IC95\%) }\end{array}$ & $\begin{array}{c}\text { RR } \\
\text { (IC95\%) }\end{array}$ \\
\hline Mortalidad & $0,99(0,88$ a 1,11$)$ & $1,04(0,91$ a 1,19$)$ & $1,07(1,0$ a 1,14$)$ & $1,10(0,98$ a 1,24$)$ \\
\hline Enfermedad Cardiovascular & $0,88(0,79$ a 0,97$)$ & $1,13(0,99$ a 1,28$)$ & $1,18(1,08$ a 1,29$)$ & $1,0(0,72$ a 1,38$)$ \\
\hline Eventos cerebrovasculares & $0,80(0,66$ a 0,96$)$ & $1,17(0,65$ a 2,09$)$ & $1,24(1,11$ a 1,4$)$ & $1,30(1,11$ a 1,53$)$ \\
\hline Enfermedad coronaria & $0,93(0,81$ a 1,07$)$ & $1,12(0,82$ a 1,54$)$ & $1,05(0,96$ a 1,15$)$ & $0,90(0,76$ a 1,06$)$ \\
\hline Discontinuación del tratamiento por eventos adversos & $3,38(0,82$ a 13,95$)$ & $1,69(0,95$ a 3,00$)$ & $1,20(0,71$ a 2,04$)$ & $1,41(1,29$ a 1,54$)$ \\
\hline
\end{tabular}

SRAA: sistema renina-angiotensina-aldosterona. RR: Riesgo relativo. IC 95\%: intervalo de confianza del 95\%.

\section{Conclusiones}

La mayoría de los ECA con betabloqueantes como tratamiento inicial para la HTA tuvieron un alto riesgo de sesgo. El atenolol fue el BB más utilizado. Iniciar tratamiento en HTA con BB conduce a reducciones modestas de eventos cardiovasculares y a poco o ningún efecto sobre la mortalidad, estos efectos son inferiores a los de otros fármacos antihipertensivos y la probabilidad de suspender el tratamiento por efectos secundarios es mayor.

Fuentes de Financiamiento: apoyo parcial del South African Medical Research Council y National Research Foundation of South Africa y financiación del Effective Health Care Research Consortium (Grant: 5242).

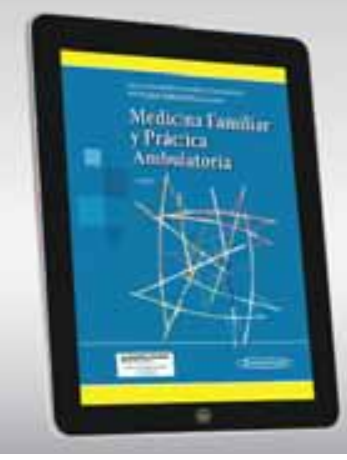

Servicio de Medicina Familiar y Comunitaria del Hospital Italiano de Buenos Aires

\section{Medicina Familiar y Práctica Ambulatoria}

3a edición

eBook

Papel

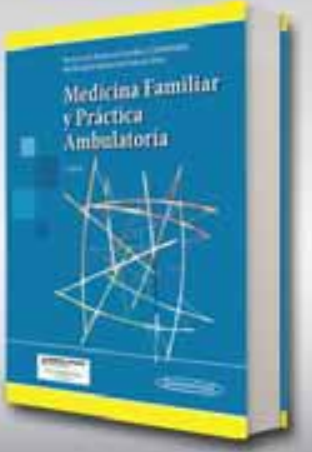




\section{Comentario}

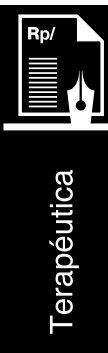

Los resultados del presente meta-análisis complementan los datos de estudios previos donde los BB, como tratamiento de primera línea en HTA, fueron inferiores a otros antihipertensivos en morbilidad y mortalidad, e inclusive mostraban mayor riesgo de eventos cerebrovasculares ${ }^{1}$. Por este motivo las guías más recientes excluyen a los betabloqueantes como tratamiento inicial en pacientes hipertensos ${ }^{2}$.

Existe gran diversidad en el grupo de los betabloqueantes en cuanto a su biodisponibilidad, cardio-selectividad y efectos hemo-dinámicos (vasodilatadores vs no vasodilatadores). La principal crítica a los ECA es el uso de atenolol (BB no vasodilatador), cuyos efectos adversos (insulino-resistencia, dislipidemia y nuevo comienzo de diabetes) pueden contribuir al menor beneficio observado ${ }^{3}$. En cambio los BB vasodilatadores (carvedilol, nebivolol) reducen la resistencia vascular periférica y tienen un efecto neutro sobre el metabolismo de la glucosa y de los lípidos. Este metaanálisis no evaluó subclases de $\mathrm{BB}$ y sus resultados no pueden extenderse a los $\mathrm{BB}$ vasodilatadores. A diferencia de los pacientes con infarto de miocardio e insuficiencia cardíaca, la disminución de la frecuencia cardíaca asociada a BB aumentó el riesgo de eventos cardiovasculares y muerte en hipertensos ${ }^{4}$. Estos hallazgos podrían explicarse por un aumento en la presión aórtica central y/o la presión del pulso secundaria al descenso de la frecuencia cardiaca, como los observados en el estudio CAFE ${ }^{5}$.

Estudios previos mostraron una reducción en la morbimortalidad cardiovascular en los pacientes más jóvenes pero no así en pacientes de mayor edad 6 . El efecto diferencial sobre la edad aún no está resuelto.

La indicación actual de BB se basa en las co-morbilidades de los pacientes hipertensos, así los que más se benefician con su uso son aquellos con HTA y angor coronario, hipertensos postIAM, insuficiencia cardíaca, taquiarritmias o disección aórtica. En hipertensos no complicados solo como tratamiento combinado son drogas de tercera o cuarta opción, o ante mecanismo fisiopatológicos de activación simpática subyacente.

\section{Conclusiones del comentador}

El atenolol no es de primera elección en el paciente hipertenso. Los BB están reservados ante la presencia de comorbilidades (enfermedad coronaria o arritmias). Son necesarios ECA que evalúen diferentes subtipos de BB, y los efectos en jóvenes y ancianos en la morbi-mortalidad.

Paula Cuffaro [ Sección Hipertensión Arterial, Servicio de Clínica Médica del Hospital Italiano de Buenos Aires. paula.cuffaro@hospitalitaliano.org.ar ]

Cuffaro P. Los betabloqueantes no son fármacos de primera línea en el tratamiento de la hipertensión arterial. Evid Act Pract Ambul. 2017;20(3):JuñSep. 69-70. Comentado de: Wiysonge CS y col. Beta-blockers for hypertension. Cochrane Database of Systematic Reviews (2017), No1.Art. No:CD002003. PMID: 28107561

\section{Referencias bibliográficas}

1. Lindholm LH, y col. Should beta blockers remain first choice in the treatment of primary hypertension? A meta-analysis. Lancet (London, England). $2005 ; 366(9496): 1545-1553$. 2. Leung AA, y col. Hypertension Canada's 2017 Guidelines for Diagnosis, Risk Assessment, Prevention, and Treatment of Hypertension in AdultsCanadian Journal of Cardiology 33 (2017) 557e576.

3. Bangalore S, y col. A meta-analysis of 94,492 patients with hypertension treated with beta blockers to determine the risk of new-onset diabetes mellitus. Am J Cardiol. 2007;100(8):1254-1262.

4. Bangalore S,y col. Relation of beta-blocker-induced heart rate lowering and cardioprotection in hypertension. J Am Coll Cardiol. 2008;52(18):1482-1489.

5 . Williams B, y col. Impact of heart rate on central aortic pressures and hemodynamics: analysis from the CAFE (Conduit Artery Function Evaluation) study: CAFE-Heart Rate. J Am Coll Cardiol. 2009 Aug 18;54(8):705-13.

6. Kuyper LM, y col. Atenolol vs nonatenolol a-blockers for the treatment of hypertension: a meta-analysis. Can J Cardiol. 2014;30(5 Suppl):S47-S53.

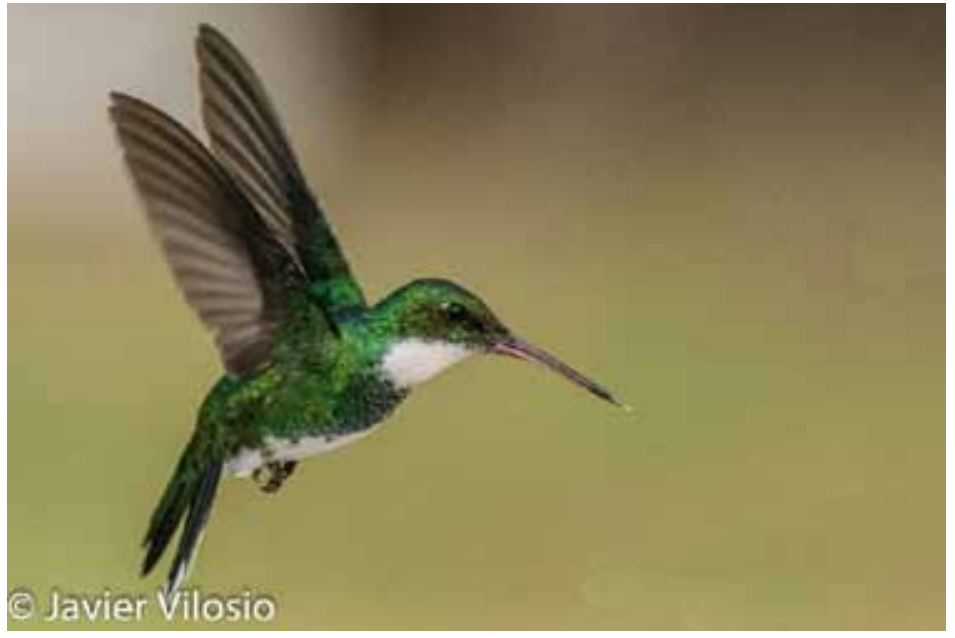

Cortesía de Javier Vilosio 